\title{
Qualidade e quantidade do leite produzido por cabras da raça Saanen submetidas a estresse por três dias consecutivos
}

\author{
[Quality and quantity of milk produced by Saanen goats submitted to stress during \\ three sequential days] \\ A.P.R. Gaiato ${ }^{1}$, T.G.F. Delgado ${ }^{2}$, J.A. Negrão ${ }^{1}$ \\ ${ }^{1}$ Universidade de São Paulo - Pirassununga, SP \\ ${ }^{2}$ UNESP-FCAV - SP \\ RESUMO
}

\begin{abstract}
Estudou-se o efeito do estresse fisiológico, via administração pontual de ACTH, sobre os níveis de cortisol e a quantidade e qualidade do leite produzido. Para tanto, 12 cabras da raça Saanen foram alocadas em dois grupos experimentais: o grupo ACTH recebeu 0,6 UI de ACTH/kg PV, e o grupo placebo recebeu solução placebo. A produção de leite, os percentuais de proteína, gordura e lactose e a contagem de células somáticas (CCS) do leite foram mensurados antes, durante e após a administração de ACTH e do placebo. Simultaneamente à administração de ACTH e placebo por três dias consecutivos, foram realizadas colheitas de sangue para mensuração dos teores de cortisol. Nos tempos -30 e zero, ambos os grupos apresentaram concentrações basais de cortisol. O aumento nos níveis de cortisol foi significativo nos tempos 60min (grupo ACTH: $59,00 \pm 5,70$ e grupo placebo: $5,23 \pm 1,37 \mathrm{ng} / \mathrm{mL}$ ) e $120 \mathrm{~min}$

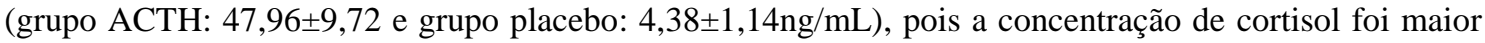
no grupo ACTH. Os valores retornaram ao basal no tempo 300min. Não houve diferenças na produção leiteira entre os grupos ACTH e placebo, tampouco de proteína, gordura, lactose e CCS do leite dos grupos, portanto o estresse induzido por três dias consecutivos não trouxe prejuízos à produção.
\end{abstract}

Palavras-chave: contagem de células somáticas, cortisol, gordura, lactose, proteína

\begin{abstract}
The experiment aimed to study the effect of physiological stress on cortisol levels, quality and quantity of milk through punctual administration of ACTH. Twelve Saanen goats were divided in two experimental

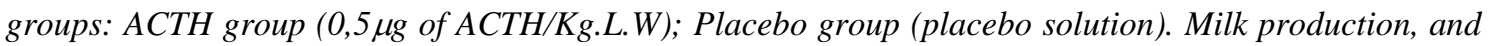
percentages of protein, fat, lactose and SCC (somatic cells counting) of the milk were analyzed before, during and after the administration of ACTH/placebo. Simultaneously to the ACTH/placebo administration and during three sequential days, blood was collected to evaluate cortisol concentrations. At times -30 and zero, both groups presented basal concentrations of cortisol. The increase of cortisol contents was significant at times 60 (group ACTH: $59.00 \pm 5.70$ and groups placebo: $5.23 \pm 1.37 \mathrm{ng} / \mathrm{mL}$ ) and 120 (group ACTH: $47.96 \pm 9.72$ and group placebo: $4.38 \pm 1,14 \mathrm{ng} / \mathrm{mL}$ ) since the cortisol content was higher on the ACTH group. The values returned to the basal level at 300 minutes. Concerning milk production, no differences were found between ACTH and placebo groups. Milk, protein, fat, lactose and SCC did not distinguish one group from another. The results indicated that the physiological stress induced during three days was not harmful to milk production and milk quality of Saanen goats.
\end{abstract}

Keywords: somatic cells counting, cortisol, fat, lactose, protein

Recebido em 29 de março de 2011

Aceito em 26 de março de 2012

E-mail: agaiato@usp.br

Apoio financeiro: FAPESP 


\section{INTRODUÇÃO}

A qualidade do leite in natura é influenciada pelo manejo, pela alimentação, pelo potencial genético dos rebanhos, além de fatores relacionados à ordenha e armazenagem do leite (Müller, 2002). O leite de alta qualidade pode ser caracterizado como um alimento livre de agentes patogênicos e outros contaminantes (resíduos de antibióticos e pesticidas), apresentando reduzida contaminação microbiana, sabor agradável, adequada composição e baixa contagem de células somáticas (Santos, 2004).

Além da qualidade do leite, a produtividade das cabras também deve ser valorizada pelos caprinocultores (Wander e Martins, 2008). Porém, alguns eventos pontuais relacionados ao manejo - parto, desmame, déficit energético podem ser associados ao estresse, promovendo manutenção de níveis elevados de cortisol (Mayer e Lefcourt, 1987; Moberg, 1987; Macuhova et al., 2002) e alterações metabólicas que reduzem a produtividade dos animais (Sapolsky et al., 2000; Pacák e Palkovits, 2001), principalmente daqueles mais produtivos.

Por esta razão, a administração exógena do ACTH, que promove a liberação de cortisol, vem sendo utilizada para mensurar a habilidade dos animais domésticos em suportar adequadamente as diferentes situações estressantes impostas ao longo de sua vida produtiva (Fulkerson e Jamieson, 1982; Negrão et al., 2004; Delgado, 2008), mensurando, assim, a habilidade dos animais em responder ao estresse (Fulkerson e Jamieson, 1982; Negrão et al., 2004).

Alguns estudos também sugerem que o estresse pode intensificar o processo de apoptose na glândula mamária durante a lactação, aumentando a contagem das células somáticas (CCS) e reduzindo, consequentemente, a produtividade dos animais (Jeng Su et al., 2002; Stefanon et al., 2002; Tian et al., 2005). Nesse contexto, a produtividade e a qualidade do leite são indicadores da evolução do tecido epitelial e da saúde da glândula mamária (Burvenich et al., 1994; Paape et al., 2002).

Desta forma, o presente experimento teve por objetivo estudar o efeito do estresse fisiológico, via administração pontual de ACTH durante três dias consecutivos, sobre os níveis de cortisol e a quantidade e qualidade do leite produzido por cabras Saanen.

\section{MATERIAL E MÉTODOS}

O experimento foi realizado com 12 cabras lactantes da raça Saanen, que permaneceram alocadas em baias com cobertura e piso ripado, tendo acesso a solário com piso de concreto. Como alimento volumoso, receberam silagem, e como concentrado uma mistura de milho, casca de soja e soja extrusada, além de sal vitamínicomineral. Concentrado e volumoso foram fornecidos na razão de 30:70 e apresentaram 47, $8 \%$ de MS; $9,9 \%$ de PB; $4,1 \%$ de EE; $7,9 \%$ de MM; 36,9\% de FDN e $22,0 \%$ de FDA. As cabras também tinham livre acesso à água em bebedouros providos de boia. Assim, todas as cabras experimentais receberam uma dieta semelhante, ajustada de acordo com o AFRC (Technical ..., 1997) em função da idade, do peso vivo, da categoria das fêmeas e da produção leiteira.

Todas as cabras experimentais foram ordenhadas uma vez por dia, com a ordenhadeira regulada para manter vácuo de $48 \mathrm{KPa}$ e taxa de pulsação de 120 ciclos/minuto. As 12 cabras lactantes foram subdivididas casualmente em dois grupos: seis cabras receberam, via intravenosa, 0,6 UI de ACTH/kg PV (Sigma, Saint Louis, Missouri, USA), para se avaliar a influência do estresse pontual por três dias consecutivos (grupo ACTH) durante a lactação (Fulkerson e Jamieson, 1982); as outras seis cabras experimentais receberam, também por via intravenosa, uma solução fisiológica (grupo placebo).

A colheita de sangue coincidia com a administração de ACTH (ou placebo) e foi executada com início às $12 \mathrm{~h} 00 \mathrm{~min}$, nos seguintes tempos de coleta composta: -30, zero (logo antes da aplicação de ACTH ou placebo), 60, 120 e 300 minutos. As amostras de sangue foram obtidas por meio da punção da veia jugular da cabra em tubos heparinizados. Após colheita, os tubos eram mantidos em gelo. Logo após, o sangue era centrifugado a $3000 \mathrm{rpm}$, a $4^{\circ} \mathrm{C}$, durante 17 minutos, e o plasma obtido era acondicionado em tubos e estocado a $-20^{\circ} \mathrm{C}$ para posterior análise e monitoramento dos níveis de cortisol. 
O plasma descongelado era utilizado para as análises de cortisol, por meio de kits imunoenzimáticos (Diagnostic Systems Laboratory Inc, Webster, Texas), executadas no Laboratório de Fisiologia Animal da FZEA/USP. A leitura dos resultados foi feita em leitor de microplacas do tipo Elisa (Multiscan MS Labsystem, Tiilitie, Vantaa, Finland), utilizando comprimento de onda de $450 \mathrm{~nm}$, baseado em uma curva padrão de sete pontos, com concentrações variando de 0,5 a $60 \mathrm{ng} / \mathrm{mL}$ de cortisol.

A produção leiteira das cabras foi mensurada diariamente, pois a ordenhadeira possui copos coletores individuais do tipo proveta. Para análise da qualidade do leite (proteína, gordura e lactose), o leite foi colhido durante 65 dias: 31 dias antes, três dias durante e 31 dias após a aplicação de ACTH/placebo. Já para a contagem de células somáticas (CCS), o leite foi colhido durante 41 dias: 19 dias antes, três dias durante e 19 dias após a aplicação de ACTH/placebo.

As lâminas para CCS foram confeccionadas e coradas com azul de toluidina para contagem microscópica direta (Prescott e Breed, 1910). Para contagem do número de células, foram observados 60 campos em cada $\mathrm{cm}^{2}$, utilizandose microscópio óptico comum com objetiva de imersão. Cada amostra foi feita em duplicata. O resultado das contagens de cada amostra foi obtido calculando-se a média dos campos multiplicada pelo fator do microscópio, obtendose a quantidade de células $/ \mathrm{mL}$ de leite. Os resultados da CCS foram convertidos para seus valores logaritmos (base 10) para análise estatística.

A maior alíquota do leite foi armazenada em frascos contendo pastilhas do conservante bronopol (2-bromo-2nitropropane-1,3-diol) para posterior análise de proteína, gordura e lactose, determinadas por infravermelho (Bentley Instruments, Inc. 2000).

O delineamento experimental foi inteiramente ao acaso, com dois grupos - ACTH e placebo - e seis animais por grupo. Foram feitas colheitas de sangue de medidas repetidas em cinco ocasiões, -30, zero, 60, 120 e 300 minutos em cada animal, durante três dias consecutivos.
Para análise estatística da produção de leite, CCS, proteína, gordura e lactose do leite e cortisol, o modelo utilizado incluiu o efeito de grupo, de tempo, de dia de colheita e a interação ACTH $x$ Tempo. Em todas as análises foi usado o proc mixed do SAS, com o desdobramento da interação visando estudar a diferença entre as respostas médias dos dois grupos em cada uma das ocasiões. Neste processo utilizou-se uma estrutura de covariâncias que melhor explicou a possível correlação existente entre as medidas repetidas nos mesmos animais e a possível heterogeneidade das variâncias nas diferentes ocasiões.

\section{RESULTADOS E DISCUSSÃO}

No tempo -30 - meia hora antes da aplicação de ACTH/placebo - e no tempo zero - momento anterior à aplicação -, ambos os grupos apresentaram concentrações basais de cortisol semelhantes (Fig. 1). A diferença entre os grupos foi observada 60 minutos após a aplicação do hormônio, pois a concentração plasmática de cortisol foi maior no grupo ACTH quando comparado ao grupo placebo, respectivamente, $59,00 \pm 5,70 \mathrm{ng} / \mathrm{mL}$ e $5,23 \pm 1,37 \mathrm{ng} / \mathrm{mL}$.

No tempo 120min, a concentração de cortisol ainda se encontrava em níveis significativamente altos no grupo que recebeu ACTH: $47,96 \pm 9,72 \mathrm{ng} / \mathrm{mL}$, versus $4,38 \pm 1,14 \mathrm{ng} / \mathrm{mL}$ do grupo placebo. Entretanto, após 300 minutos da aplicação do ACTH, os valores de cortisol retornaram ao considerado basal para ambos os grupos. Desse modo, o grupo que recebeu ACTH apresentou um mesmo modelo de liberação de cortisol nos três dias de desafio, com valores de cortisol superiores aos do grupo placebo, o que indica que houve estímulo da adrenal pelo ACTH (Escobar et al., 1998; Negrão et al., 2004; Delgado, 2008). Tanto o perfil quanto o aumento nos níveis de cortisol mensurados nas cabras experimentais que receberam ACTH foram próximos àqueles observados após a indução de diferentes agentes estressores, seja em cabras (Escobar et al., 1998; Duvaux-Ponter et al., 2003; Delgado, 2008; Canaes et al., 2009; Canaes e Negrão, 2009), em ovelhas (Fulkerson e Jamieson, 1982; Marnet e Negrão, 2000) ou em vacas (Tancin et al., 1995; Rushen et al., 2001; Negrão et al., 2004; Negrão, 2008). 


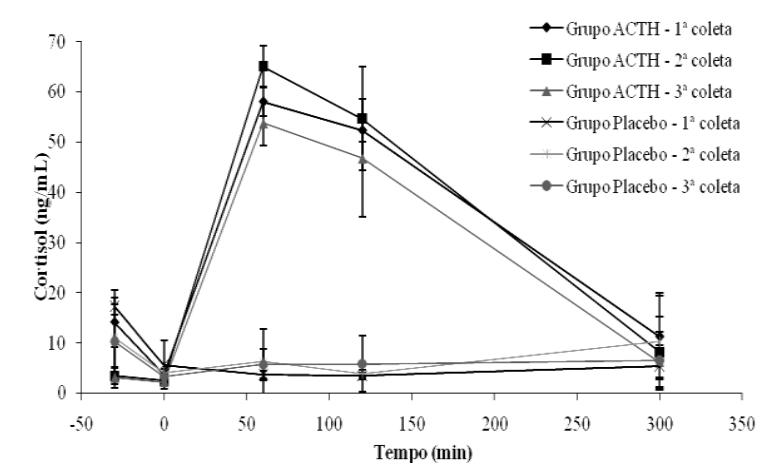

Figura 1. Teores plasmáticos médios de cortisol de ambos os grupos (ACTH e placebo) durante o período experimental em três dias consecutivos de aplicação de ACTH/placebo.

Não foram observadas diferenças entre tratamentos para produção de leite (Fig. 2), o que indica que cabras estressadas pontualmente por três dias consecutivos não diminuíram a produção de leite em função do aumento dos

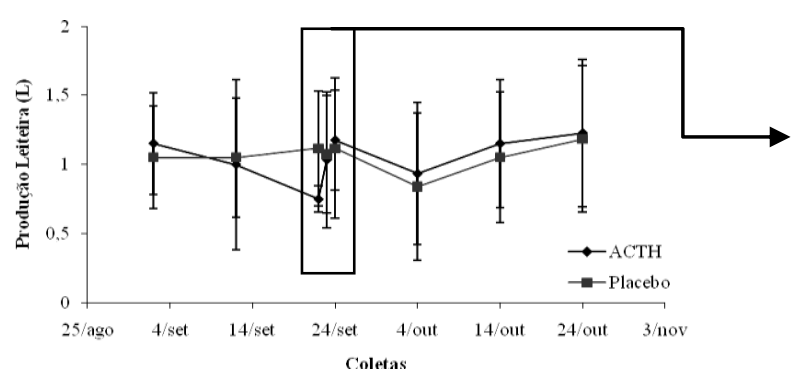

níveis de cortisol. Outros estudos também não demonstraram o efeito do estresse pontual sobre a produção leiteira (Tancin et al., 1995; Rushen et al., 2001; Delgado, 2008; Canaes e Negrão, 2009).

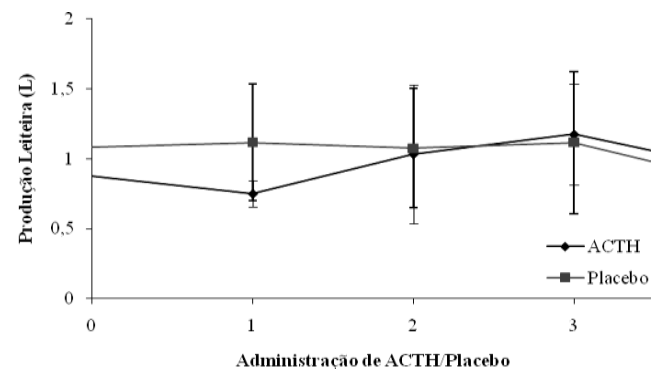

Figura 2. Produção leiteira média de ambos os grupos (ACTH/placebo) durante 65 dias: 31 dias antes do tratamento, três dias durante e 31 dias depois.

Também não houve diferenças na distribuição dos valores médios de proteína no leite durante o estresse pontual por três dias consecutivos. Cabras submetidas ao estresse via transporte e com aumento dos níveis de cortisol também não apresentaram mudanças nos teores de proteína do leite (Canaes e Negrão, 2009). No período experimental, os teores de proteína variaram de
$3,15 \pm 0,09$ e $3,23 \pm 0,07 \%$ para cabras do grupo placebo e ACTH, respectivamente (Fig. 3). Valores observados no presente estudo estão entre 2,3 e 4,2\%, faixa considerada normal para cabras (Karin e Lofti, 1987; Espie e Mullan, 1990), pois a proteína é um dos componentes do leite que apresenta concentração mais estável no leite (Queiroga et al., 2007).

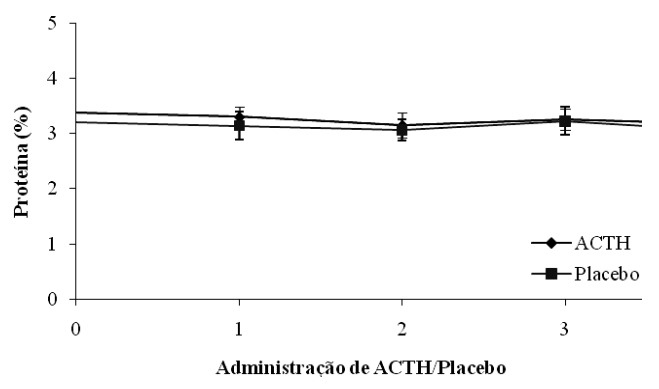

Figura 3. Teores médios de proteína do leite de cabras de ambos os grupos (ACTH/placebo) durante 65 dias: 31 dias antes do tratamento, três dias durante e 31 dias depois. 
Não foram observadas diferenças entre os grupos, e os teores médios de gordura do leite

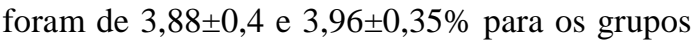
placebo e ACTH, respectivamente (Fig. 4). Apesar de a gordura ser o componente que sofre maior variação (Gomes et al., 2004; Brito et al.,
2006), os percentuais médios de gordura do leite mensurados no presente estudo estão situados entre 3,5 e $4,5 \%$, sendo considerados normais para cabras leiteiras (Brasil et al., 2000; Barbosa et al., 2002).

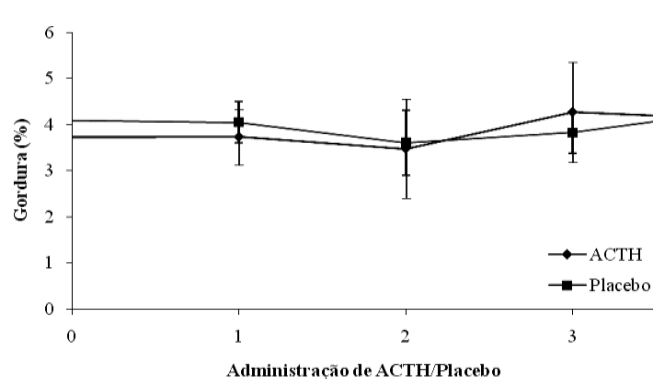

Figura 4. Teores médios de gordura do leite de cabras de ambos os grupos (ACTH/placebo) durante 65 dias: 31 dias antes do tratamento, três dias durante e 31 dias depois.

Também não houve diferenças significativas nos teores de lactose do leite entre os grupos experimentais, sendo que os teores de lactose variaram de 4,46 $\pm 0,12$ para o grupo placebo e $4,39 \pm 0,09 \%$ para o grupo ACTH (Fig. 5). Por ser precursora da síntese láctea, a lactose (glicose + galactose) é o componente mais estável do leite, com isso, sua concentração sofre poucas

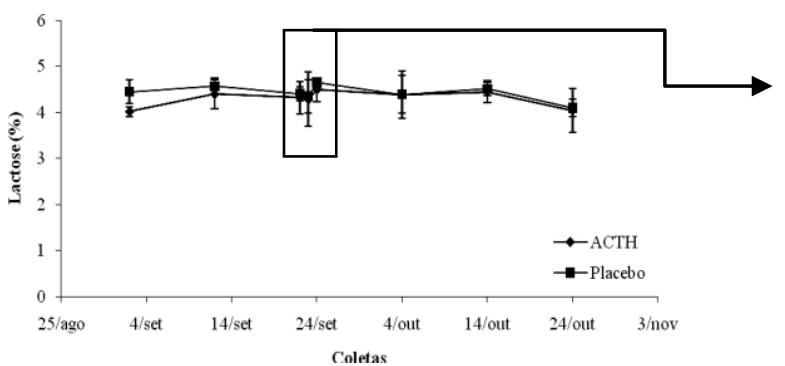

alterações durante a lactação. Assim, mesmo com a maior disponibilidade de glicose no sangue e o aumento na síntese de lactose, não haverá aumento da concentração deste componente no leite e sim aumento do volume de leite produzido (Harmon, 1994; González, 2003).

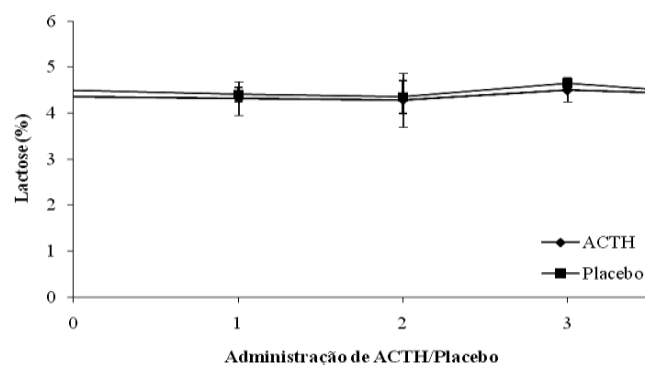

Figura 5. Teores médios de lactose do leite de cabras de ambos os grupos (ACTH/placebo) durante 65 dias: 31 dias antes do tratamento, três dias durante e 31 dias depois.

Não foram observadas diferenças significativas na CCS entre os grupos experimentais (Fig. 6). Os valores de CCS mensurados estão situados na faixa indicada para cabras sadias (ausência de mastite): 270 a $2000 \times 10^{3} / \mathrm{mL}$ de leite (Galiero e Morena, 2000; Paape et al., 2001). Quando a CCS foi convertida em valor de $\log$, o grupo placebo apresentou média de 5,93 $\pm 0,01$ de valor de $\log$, enquanto o grupo placebo apresentou média de $6,10 \pm 0,14$ de valor de log durante o período experimental, valores também considerados normais (Zeng e Escobar, 1996; Andrade et al., 2001; Olechnowicz e Sobek,
2008). O fato de a CCS não ter sido influenciada pela administração de ACTH por três dias consecutivos não era o esperado, já que o estresse supostamente elevaria a taxa de apoptose das células epiteliais (Boutinaud et al., 2004; Tian et al., 2005), o que diminuiria a produção (Capuco et al., 1997; Caldoncelli et al., 2002). Porém, a CCS permaneceu estável no presente experimento, o que indica que cabras estressadas pontualmente por três dias consecutivos não diminuem a qualidade e a produtividade do leite devido ao aumento dos níveis de cortisol. 

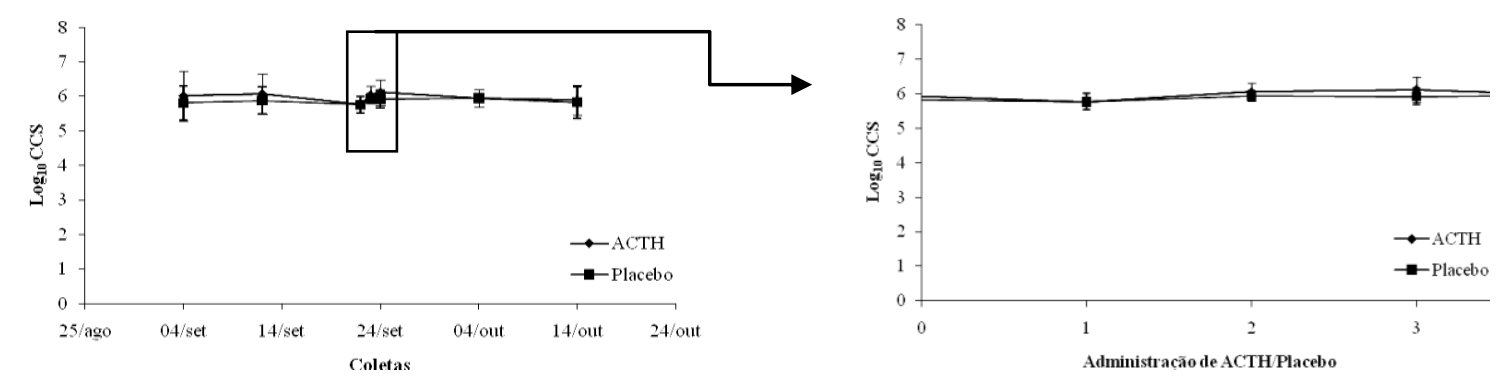

Figura 6. Média do logaritmo da contagem de células somáticas do leite de cabras de ambos os grupos (ACTH/placebo) durante 41 dias: 19 dias antes do tratamento, três dias durante e 19 dias depois.

Não houve qualquer tipo de correlação significativa $(\mathrm{P}<0.05)$ entre níveis de cortisol e quantidade de leite $(\mathrm{r}=-0,08)$, proteína $(\mathrm{r}=-0,39)$, gordura $(\mathrm{r}=0,05)$, lactose $(\mathrm{r}=-0,14)$ e $\log$ de CCS $(\mathrm{r}=-0,21)$. Caroprese et al. (2010), contudo, verificaram que ovelhas lactantes isoladas apresentaram elevação dos teores de cortisol, diminuição da produção leiteira e aumento da CCS. Assim, embora as cabras que receberam ACTH tenham liberado significativamente mais cortisol que as cabras que receberam a solução placebo, não houve queda na qualidade tampouco na quantidade de leite produzido, portanto o estresse induzido por três dias consecutivos não causou efeito negativo às cabras Saanen.

\section{REFERÊNCIAS}

ANDRADE, P.V.D.; SOUZA, M.R.; BORGES, I.; PENNA, C.F.A.M. Contagem de células somáticas em leite de cabra. Arq. Bras. Med. Vet. Zootec., v.53, p.396-400, 2001.

BARBOSA, P.G.; GONÇALVES, H.C.; WECHSLER, F.S. et al. Uso da somatotropina bovina recombinante - rbST como alternativa para a produção de leite de cabra na entressafra. Rev. Bras. Zootec., v.31, p.2011-2023, 2002.

BOUTINAUD, M.; GUINARD-FLAMENT, J.; JAMMES, $H$. The number and activity of mammary epithelial cells, determining factors for milk production. Reprod. Nutr. Dev., v.44, p.499-508, 2004.

BRASIL, L.H.A.; WECHESLER, F.S.; BACCARI Jr., F. et al. Efeitos do estresse térmico sobre a produção, composição química do leite e respostas termorreguladoras de cabras da raça Alpina. Rev. Bras. Zootec., v.29, p.1632-1641, 2000.
BRITO, M.A.; DIAZ GONZALEZ, F.H.; RIBEIRO, L.A.O. et al. Composição do sangue e do leite em ovinos leiteiros do sul do Brasil: variações na gestação e na lactação. Cienc. Rural, v.36, p.942-948, 2006.

BURVENICH, C.; PAAPE, M.J.; HILL, A.W. et al. Role of the neutrophil leukocyte in the local and systemic reactions during experimentally induced $E$. coli mastitis in cows immediately after calving. Vet. $Q .$, v.16, p.45-50, 1994.

CALDONCELLI, F.F.; FARIAS, R.E.; GUERRA, M.O. et al. Apoptose na involução da glândula mamária. Rev. Bras. Mastol., v.12, p.39-44, 2002.

CANAES, T.S.; NEGRÃO, J.A. Aspectos fisiológicos, comportamentais e produtivos de cabras Alpinas submetidas ao transporte. Rev. Bras. Zootec., v.38, p.893-897, 2009.

CANAES, T.S.; NEGRÃO, J.A.; PAIVA, F.A. et al. Influência do transporte e mudança do local de manejo nas variáveis fisiológicas e produtivas de cabras Alpinas. Arq. Bras. Med. Vet. Zootec., v.61, p.935940, 2009.

CAPUCO, A.V.; AKERS, R.M.; SMITH, J.J. Mammary growth in Holstein cows during the dry period: Quantification of nucleic acids and histology. J. Dairy Sci., v.80, p.477-487, 1997.

CAROPRESE, M.; ALBENZIO, M; MARZANO, A. et al. Relationship between cortisol response to stress and behavior, immune profile, and production performance of dairy ewes. J. Dairy Sci., v.93, p.23952403, 2010.

DELGADO, T.F.G. Produção leiteira em cabras da raça Saanen: Influência dos hormônios Cortisol $e$ IGF-I. 2008. 91f. Dissertação (Mestrado em Zootecnia) - Faculdade de Ciências Agrárias e Veterinárias, Universidade Estadual Paulista, Jaboticabal, SP.

DUVAUX-PONTER, C.; ROUSSEL, S.; TESSIER, J. et al. Physiological effects of repeated transport in pregnant goats and their offspring. Anim. Res., v.52, p.553-566, 2003. 
ESCOBAR, C.J.; BASRUR, P.K.; GARTLEY, C.; LIPTRAP, R.M. A comparison of the adrenal cortical response to ACTH stimulation in Angora and nonAngora goats. Vet. Res. Commun., v.22, p.119-129, 1998.

ESPIE, W.E.; MULLAN, W.M.A. Microbiological aspects of goat milk in Northern Ireland. Milchwissenschaft, v.45, p.361-362, 1990.

FULKERSON, W.J.; JAMIESON, P.A. Pattern of cortisol release in sheep following administration of synthetic ACTH or imposition of various stressor agents. Aust. J. Biol. Sci., v.35, p.215-222, 1982.

GALIERO, G.; MORENA, C. The meaning of the somatic cell count in buffalo milk. Bubalus bubalis, n.4, p.26-27, 2000.

GOMES, V.; DELLA LIBERA, A.M.M.P.; MADUREIRA, K.M.; ARAÚJO, W.P. Influência do estágio de lactação na composição do leite de cabras (Capra hircus). Braz. J. Vet. Res. Anim. Sci., v.41, p.339-342, 2004.

GONZÁLEZ, F.H.D. Indicadores metabóliconutricionais do leite. In: GONZÁLEZ, F.H.D.; CAMPOS, R. Simpósio de Patologia Clínica Veterinária da Região Sul do Brasil, 1., 2003, Porto Alegre. Anais... Porto Alegre: Universidade Federal do Rio Grande do Sul, 2003. p.31-47.

HARMON, R.J. Physiology of mastitis and factors affecting somatic cell count. J. Dairy Sci., v.77, p.2103-2112, 1994.

JENG SU, W.; JU CHANG, C.; CHENG PEH, H. et al. Apoptosis and oxidative stress infiltrated neutrophils obtained from mammary glands of goats during various stages of lactation. Am. J. Vet. Res., v.63, p.241-246, 2002.

KARIN, J.; LOFTI, A. Studies on the milk composition of crossbreed Saanen goats. J. V. Fac. Univ. Teheran, Iran, v.42, p.5-13, 1987.

MACUHOVA, J.; TANCIN V.; KRAETZL W.D. et al. Inhibition of oxytocin release during repeated milking in unfamiliar surroundings: The importance of opioids and adrenal cortex sensitivity. J. Dairy Res., v.69, p.63-73, 2002.

MARNET, P.G.; NEGRÃO J.A. The effect of a mixed-management system on the release of oxytocin, prolactin, and cortisol in ewes during sucking and machine milking. Reprod. Nutr. Dev., v.40, p.271-281, 2000.

MAYER, H.K.; LEFCOURT, A.M. Failure of cortisol injected prior to milking to inhibit milk ejection in dairy cattle. J. Dairy Res., v.54, p.173-177, 1987.

MOBERG, G.P. A model for assessing the impact of behavioral stress on domestic animals. J. Anim. Sci., v.65, p.1228-1235, 1987.
MÜLLER, E.E. Qualidade do leite, células somáticas e prevenção da mastite. In: Leite: Simpósio sobre Sustentabilidade da Pecuária Leiteira na Região Sul do Brasil, 2., 2002, Maringá. Anais... Maringá: Universidade Estadual de Maringá, 2002. p.206-217.

NEGRÃO, J.A.; PORCIONATO, M.A.F.; De PASSILLÉ, A.M.; RUSHEN, J. Cortisol in saliva and plasma of cattle after ACTH administration and milking. J. Dairy Sci., v.87, p.1713-1718, 2004.

NEGRÃO, J.A. Hormone release and behavior during suckling and milking in Gir, Gir x Holstein cow. $J$. Anim. Sci., v.86, p.21-26, 2008.

OLECHNOWICZ, J.; SOBEK, Z. Factors of variation influencing production level, SCC and basic milk composition in dairy goats. J. Anim. Feed Sci., v.17, p.41-49, 2008.

PAAPE, M.J.; POUTREL, B.; CONTRERAS, A. $e t$ al. Milk somatic cells and lactation in small ruminants. J. Dairy Sci., v.84, Suppl., p.237-244, 2001.

PAAPE, M.J.; MEHZARD, J.; ZHAO, X. et al. Defense of the bovine mammary gland by polymorphonuclear neutrophil leukocytes. $J$. Mammary Gland Biol. Neoplasia, v.7, p.109-121, 2002.

PACÁK, K.; PALKOVITS, M. Stressor specificity of central neuroendocrine response: implications for stress-related disorders. Endocr. Rev., v.22, p.502-548, 2001.

PRESCOTT, S.C.; BREED. R.S. The determination of the number of body cells in milk by a direct method. $J$. Infect. Dis., v.7, p.632-640, 1910.

QUEIROGA, R.C.R.E.; COSTA, R.G.; BISCONTINI, T.M.B. et al. Influência do manejo do rebanho, das condições higiênicas da ordenha e da fase de lactação na composição química do leite de cabras Saanen. Rev. Bras. Zootec., v.36, p.430-437, 2007.

RUSHEN, J.; MUNKSGAARD, L.; MARNET, P.G.; DE PASSILÉ, A.M. Human contact and the effects of acute stress on cows at milking. Appl. Anim. Behav. Sci., v.73, p.1-14, 2001.

SANTOS, M.V. Aspectos não microbiológicos afetando a qualidade do leite. In: DURR, J.W.; CARVALHO, M.P.; SANTOS, M.V. O compromisso com a qualidade do leite no Brasil. Passo Fundo: UPF editora, 2004. p.269-283.

SAPOLSKY, R.M.; ROMERO, L.M.; MUNCK, A.U. How do glucocorticoids influence stress response? Integrating permissive, supressive, stimulatory and preparative actions. Endocr. Rev., v.21, p.55-89, 2000.

STEFANON, B.; COLITTI, M.; GIANFRANCO, G. et al. Mammary apoptosis and lactation persistency in dairy animals. J. Dairy Res., v.69, p.37-52, 2002. 


\section{Gaiato et al.}

TIAN, S.Z.; CHANG, C.J.; CHIANG, C.C. et al. Comparison of morphology, viability, and function between blood and milk neutrophils from peak lactating goats. Can. J. Vet. Res., v.69, p.39-45, 2005.

TANCIN, V.; HARCEK, L.; BROUCEK, J. et al. Effects of suckling during early lactation and changeover to machine milking on plasma oxytocin and cortisol levels and milking characteristics in Holstein cows. J. Dairy Res., v.62, p.249-256, 1995.
TECHNICAL Committee on Responses to Nutrients, Report n.10. AFRC. The nutrition of goats. Nutrit. Abst. Rev. (series B), v.67, p.765-830, 1997.

WANDER, A.E.; MARTINS, E.C. Viabilidade econômica da caprinocultura leiteira. Anuário Brasileiro de Caprinos \& Ovinos. Uberaba: Agropecuária Tropical, 2008. 15p.

ZENG, S.S.; ESCOBAR, E.N. Effect of breed and milking method on somatic cell count, standard plate count and composition of goat milk. Small Rum. Res., v.19, p.169-175, 1996. 